\title{
Unsupervised Learning in Reservoir Computing: Modeling Hippocampal Place Cells for Small Mobile Robots
}

\author{
Eric A. Antonelo* and Benjamin Schrauwen \\ Electronics and Information Systems Department, Ghent University, \\ Sint Pietersnieuwstraat 41, 9000 Ghent, Belgium \\ \{eric.antonelo, benjamin.schrauwen\}@elis.ugent.be \\ http://reslab.elis.ugent.be
}

\begin{abstract}
Biological systems (e.g., rats) have efficient and robust localization abilities provided by the so called, place cells, which are found in the hippocampus of rodents and primates (these cells encode locations of the animal's environment). This work seeks to model these place cells by employing three (biologically plausible) techniques: Reservoir Computing (RC), Slow Feature Analysis (SFA), and Independent Component Analysis (ICA). The proposed architecture is composed of three layers, where the bottom layer is a dynamic reservoir of recurrent nodes with fixed weights. The upper layers (SFA and ICA) provides a self-organized formation of place cells, learned in an unsupervised way. Experiments show that a simulated mobile robot with 17 noisy short-range distance sensors is able to self-localize in its environment with the proposed architecture, forming a spatial representation which is dependent on the robot direction.
\end{abstract}

Key words: reservoir computing, slow feature analysis, place cells

\section{Introduction}

Animals or robots should be able to efficiently self-localize in their environments for learning and accomplishing cognitive intelligent behaviors. They must be able to seek targets (energy resources) and accomplish important tasks in a efficient way. In this context, the ability to self-localize is clearly needed.

Standard robot localization systems are designed mostly by probabilistic methods which can perform SLAM (Simultaneous Localization And Mapping) under suitable assumptions [1] and are usually built for robots having highresolution expensive laser scanners. Biologically inspired systems for robot localization can be considered a competitive alternative that works also for small mobile robots. Robustness, learning and low computation time are some characteristics of these biological inspired systems. Most systems are based on visual input from camera [2-4] and models hippocampal place cells from rats [2-5].

\footnotetext{
* This work was partially supported by FWO Flanders project G.0317.05.
} 
These place cells are the main components of the spatial navigation system in rodents. Each place cell codes for a particular location of the rat's environment, presenting a peak response in the proximities of that location (the place field of that cell). Other components of the brain's spatial representation system includes head-direction cells, which encode the orientation of the animal in its environment, and grid cells, which are non-localized representations of space (having a grid-like structure of activations in space) [6]. Grid cells are found in the entorhinal cortex of rats and probably have an important role in the formation of place cells in the hippocampus [6]. Two classes of stimuli are available to place and grid cells: idiothetic and allothetic. Idiothetic input is originated from the physical body, such as proprioceptive sensors, which can be used for dead reckoning (path integration). Allothetic information is obtained from the external environment via sensors like distance sensors and camera. Dead reckoning can usually be corrected using allothetic information. The current work models place cells and to some extent, grid cells, endowing a simulated mobile robot with the capacity to self-localize in its environment through an unsupervised learning process. Reservoir Computing (RC) [7, 8], Slow Feature Analysis (SFA) [9], and Independent Component Analysis (ICA) [10] are three (biologically plausible) techniques used in this work for modeling place cells. RC is a recently introduced paradigm in Recurrent Neural Networks (RNN) where the recurrent connections are not trained at all. Only output units are trained (usually in a supervised way) while the reservoir (the RNN itself) is a randomly generated dynamic system with fixed weights [8]. RC has biological foundations as it is shown, for example, that Liquid State Machines (a type of RC) are based on the micro-column structure in the cortex [11]. Furthermore, works such as in [12] establish a strong association between real brains and reservoirs. SFA is another recently proposed method to extract invariant or slowing varying features from input data [9]. Similarly to [3], we use SFA to model grid cells. While they use several SFA layers and high-dimensional input from a camera, we use only few noisy distance sensors and a RC-SFA based architecture.

This work proposes a general architecture based on reservoir computing and slow feature analysis (RC-SFA). While SFA provides an unsupervised learning mechanism for reservoirs, the latter provides short-term memory to SFA-based systems. This powerful combination can also be used in more general applications (such as speech recognition and robot behavior modeling). The proposed architecture is used here for autonomous map learning by modeling hippocampal place cells. A mobile robot with 17 short-range distance sensors is sufficient for generating a rather accurate spatial representation of maze-like environments without using proprioceptive information (odometry). The robot, in this way, autonomously learns to self-localize in its environment.

\section{Methods}

\subsection{Reservoir Computing}

The first layer of the RC-SFA architecture consists of a randomly created recurrent neural network, that is, the reservoir. This network is composed of sigmoidal 
neurons and is modeled by the following state update equation [8]:

$$
\mathbf{x}(t+1)=f\left((1-\alpha) \mathbf{x}(t)+\alpha\left(\mathbf{W}_{\mathrm{in}} \mathbf{u}(t)+\mathbf{W}_{\mathrm{res}} \mathbf{x}(t)\right)\right),
$$

where: $\mathbf{u}(t)$ denotes the input at time $t ; \mathbf{x}(t)$ represents the reservoir state; $\alpha$ is the leak rate [13]; and $f()=\tanh ()$ is the hyperbolic tangent activation function (the most common type of activation function used for reservoirs). The connections between the nodes of the network are represented by weight matrices: $\mathbf{W}_{\text {in }}$ is the connection matrix from input to reservoir and $\mathbf{W}_{\text {res }}$ represents the recurrent connections between internal nodes. The initial state of the dynamical system is $\mathbf{x}(0)=\mathbf{0}$. A standard reservoir equation (without the leak rate) is found when $\alpha=1$.

The matrices $\mathbf{W}_{\text {in }}$ and $\mathbf{W}_{\text {res }}$ are fixed and randomly created at the beginning. Each element of the connection matrix $\mathbf{W}_{\text {res }}$ is drawn from a normal distribution with mean 0 and variance 1 . The randomly created $\mathbf{W}_{\text {res }}$ matrix is rescaled such that the system is stable and the reservoir has the echo state property (i.e., it has a fading memory [8]). This can be accomplished by rescaling the matrix so that the spectral radius $\left|\lambda_{\max }\right|$ (the largest absolute eigenvalue) of the linearized system is smaller than one [8]. Standard settings of $\left|\lambda_{\max }\right|$ lie in a range between 0.7 and 0.98 [8]. In this work we scale all reservoirs $\left(\mathbf{W}_{\text {res }}\right)$ to a spectral radius of $\left|\lambda_{\max }\right|=0.9$ which is an arbitrarily chosen value (shown to produce good results). The initialization of $\mathbf{W}_{\text {in }}$ is given in Section 3.1.

The leak rate $\alpha$ should be in the interval $(0,1]$ and can be used to tune the dynamics of the reservoir [13]. In this way, lower leak rates slow down the reservoir, increasing its memory but decreasing its capacity for agile processing of the input signal. Higher leak rates yield fast processing of the input but low memory to hold past stimuli. Similar results can be achieved when resampling the input signal for matching the timescale of the reservoir. For instance, it might be necessary to downsample an input signal if it varies too slowly. In this work, $d_{t}$ represents the downsampling rate of the original input signal.

In this paper, the RC-SFA architecture is composed of a hierarchical network of nodes where the lower layer is the reservoir and the upper layers are composed of SFA and ICA units, respectively (Fig. 1). This hierarchical network learns in a unsupervised way (except for the reservoir whose weights $\left(\mathbf{W}_{\text {in }}\right.$ and $\mathbf{W}_{\text {res }}$ ) are kept fixed). The function of the reservoir is to map the inputs to a highdimensional dynamic space. Because of its recurrent connections, the reservoir states contain echoes of the past inputs, providing a short-term memory to our model. The SFA layer receives signals from the input nodes $\mathbf{u}(t)$ and from the reservoir nodes $\mathbf{x}(t)$. This layer generates invariant or slowly varying signals [9] which are instantaneous functions of input from previous layers (see Section 2.2). The upper-most layer is composed of ICA units which generate a sparse and local representation of the slowing varying SFA features. The following sections focus on these upper layers. Next, consider $n_{\mathrm{u}}$ as the number of inputs; $n_{\text {res }}$ as the number of neurons in the reservoir; $n_{\mathrm{sfa}}$ as the number of SFA units; and $n_{\text {ica }}$ as the number of ICA units. 


\subsection{Slow Feature Analysis}

Slow Feature Analysis (SFA) is a recently introduced algorithm that finds functions which are independent and slowly varying representations of the input [9]. SFA has also been shown to reproduce qualitative and quantitative properties of complex cells found in the primary visual cortex (V1) [14] and grid-cells from the entorhinal cortex of rats [3].

The learning task can be defined as follows. Given a high-dimensional input signal $\mathbf{x}(t)$, find a set of scalar functions $g_{i}(\mathbf{x}(t))$ so that the SFA output $y_{i}=$ $g_{i}(\mathbf{x}(t))$ varies as slowly as possible and still carries significant information. In mathematical terms [9], find output signals $y_{i}=g_{i}(\mathbf{x}(t))$ such that:

$$
\Delta\left(y_{i}\right):=\left\langle\dot{y}_{i}^{2}\right\rangle_{t} \quad \text { is minimal }
$$

under the constraints

$$
\begin{aligned}
\left\langle y_{i}\right\rangle_{t} & =0 & & \text { (zero mean) } \\
\left\langle y_{i}^{2}\right\rangle_{t} & =1 & & \text { (unit variance) } \\
\forall j<i,\left\langle y_{i} y_{j}\right\rangle_{t} & =0 & & \text { (decorrelation and order) }
\end{aligned}
$$

where $\langle.\rangle_{t}$ and $\dot{y}$ denote temporal averaging and the derivative of $y$, respectively.

Learning: Before applying the algorithm, the input signal $\mathbf{x}(t)$ is normalized to have zero mean and unit variance. In this work, we only consider the linear case $g_{i}(\mathbf{x})=\mathbf{w}^{T} \mathbf{x}$, because the reservoir is already non-linear. The SFA algorithm is as follows:

Solve the generalized eigenvalue problem:

$$
\mathbf{A W}=\mathbf{B W} \Lambda,
$$

where $\mathbf{A}:=\left\langle\dot{\mathbf{x}} \dot{\mathbf{x}}^{T}\right\rangle_{t}$ and $\mathbf{B}:=\left\langle\mathbf{x} \mathbf{x}^{T}\right\rangle_{t}$.

The eigenvectors $\mathbf{w}_{1}, \mathbf{w}_{2}, \ldots, \mathbf{w}_{n_{s f a}}$ corresponding to the ordered generalized eigenvalues $\lambda_{1} \leq \lambda_{2} \leq \ldots \leq \lambda_{n_{s f a}}$ solve the learning task, satisfying (3-5) and minimizing (2) (see [9] for more details). This algorithm is guaranteed to find the global optimum.

Architecture: The SFA layer in our architecture (Fig. 1) is denoted by $\mathbf{y}_{\text {sfa }}(t)$ :

$$
\mathbf{y}_{\text {sfa }}(t)=\mathbf{W}_{\text {sfa }} \mathbf{x}_{\text {sfa }}(t)
$$

where: $\mathbf{x}_{\mathrm{sfa}}(t)$ is the input vector at time $t$ consisting of a concatenation of input $\mathbf{u}(t)$ and reservoir states $\mathbf{x}(t)$. Note that the states $\mathbf{x}(t)$ are generated by stimulating the reservoir with the input signal $\mathbf{u}(t)$ for $t=1,2, \ldots n_{\mathrm{s}}$ by using (1), where $n_{\mathrm{s}}$ is the number of samples. The connection matrix $\mathbf{W}_{\mathrm{sfa}}$ is a $n_{\text {sfa }} \times\left(n_{\mathrm{u}}+n_{\text {res }}\right)$ matrix corresponding to the eigenvectors found by solving (6). In this work, the output signal $\mathbf{y}_{\mathrm{sfa}}(t)$ generates non-localized representations of the environment, similarly to grid cells of the entorhinal cortex of rats [6].

\subsection{Independent Component Analysis}

Independent Component Analysis (ICA) is a method used for sparse coding of input data as well as for blind source separation [10]. The ICA model assumes that a linear mixture of signals $x_{1}, x_{2} \ldots x_{n}$ can be used for finding the 
$n$ independent components or latent variables $s_{1}, s_{2} \ldots s_{n}$. The observed values $\mathbf{x}(t)=\left[x_{1}(t), x_{2}(t) \ldots x_{n}(t)\right]$ can be written as:

$$
\mathbf{x}(t)=\mathbf{A s}(t)
$$

where $\mathbf{A}$ is the mixing matrix; and $\mathbf{s}(t)=\left[s_{1}(t), s_{2}(t) \ldots s_{n}(t)\right]$ is the vector of independent components (both $\mathbf{A}$ and $\mathbf{s}(t)$ are assumed to be unknown). The vector $\mathbf{s}(t)$ can be generated after estimating matrix $\mathbf{A}$ :

$$
\mathbf{s}(t)=\mathbf{W} \mathbf{x}(t)
$$

where $\mathbf{W}$ is the inverse matrix of $\mathbf{A}$. The basic assumption for ICA is that the components $s_{i}$ are statistically independent. It is also assumed that the independent components have nongaussian distributions [10].

Learning: In this work the matrix $\mathbf{W}$ is found with the FastICA algorithm [10]. Before using ICA, the observed vector $\mathbf{x}(t)$ is preprocessed by centering (zero-mean) and whitening (decorrelation and unit variance) [10]. FastICA uses a fixed-point iteration scheme for finding the maximum of the nongaussianity of $\mathbf{w} \mathbf{x}(t)$ (where $\mathbf{w}$ is a weight vector of one neuron). The basic form of the FastICA algorithm (for one unit) is described next:

1. Initialize $\mathbf{w}$ randomly

2. Let $\mathbf{w}^{+}=E\left\{\mathbf{x} g\left(\mathbf{w}^{T} \mathbf{x}\right)\right\}-E\left\{g^{\prime}\left(\mathbf{w}^{T} \mathbf{x}\right) \mathbf{w}\right\}$

3. Let $\mathbf{w}=\mathbf{w}^{+} /\left\|\mathbf{w}^{+}\right\|$

4. Do steps 2 and 3 until convergence,

where $g$ is the derivative of a nonquadratic function $G$ (in this work, $G(u)=u^{3}$ ) (see [10] for a detailed description).

Architecture: The equation for the ICA layer is (by redefining variables):

$$
\mathbf{y}_{\text {ica }}(t)=\mathbf{W}_{\text {ica }} \mathbf{y}_{\text {sfa }}(t),
$$

where: $\mathbf{y}_{\text {sfa }}(t)$ is the input vector at time $t$ (the observed values); $\mathbf{W}_{\text {sfa }}$ is the mixing matrix $\left(n_{\text {ica }} \times n_{\text {sfa }}\right)$; and $\mathbf{y}_{\text {ica }}(t)$ is the output of the ICA layer (the independent components), which, in this work, learns to generate localized outputs which model hippocampal place cells of rats [6].

\subsection{Robot Model}

The robot model used in this work (Fig. 1) is part of the 2D SINAR simulator [15] and is described next. The robot interacts with the environment by distance and color sensors; and by one actuator which controls the movement direction (turning). Seventeen (17) sensor positions are distributed uniformly over the front of the robot (from $-90^{\circ}$ to $+90^{\circ}$ ). Each position holds two virtual sensors (for distance and color perception) [15]. The distance sensors are limited in range (i.e., they saturate for distances greater than 300 distance units (d.u.)) and are noisy (they exhibit Gaussian noise on their readings, generated from $N(0,0.01))$. A value of 0 means near some object and a value of 1 means far or nothing detected. At each iteration the robot is able to execute a direction adjustment to the left or to the right in the range $[0,15]$ degrees and the speed is constant (0.28 distance units (d.u.)/s). The SINAR controller (based on [15]) is an intelligent navigation system made of hierarchical neural networks which 
(a)

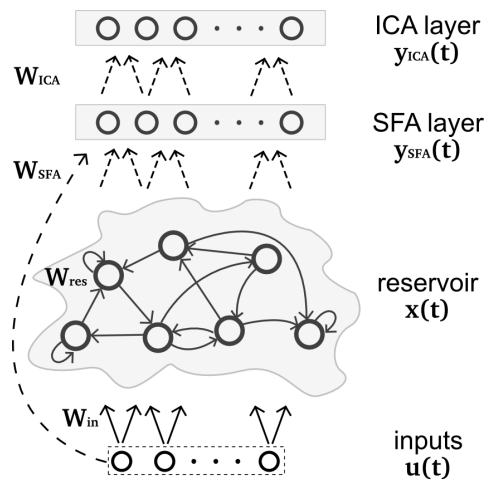

(b)

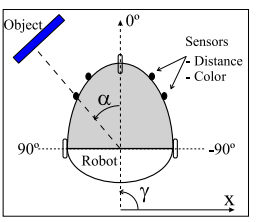

(c)

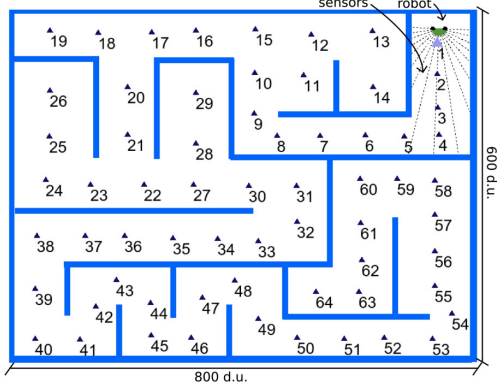

Fig. 1. (a) RC-SFA architecture. (b) Robot model. (c) Environment E1. The environment is tagged with 64 labels displayed by small triangles.

learn by interaction with the environment. After learning, the robot is able to efficiently navigate and explore environments, during which the signal $\mathbf{u}(t)$ is built by recording the 17 distance sensors of the robot.

\section{$3 \quad$ Experiments}

\subsection{Introduction}

In the following, we describe the architecture (Fig. 1) and the initialization of parameters for the experiments. The first layer of the architecture corresponds to a dynamic reservoir of 400 neurons, which provides short-term memory to our model. The second layer consists of 70 SFA units, which extracts the slow features from the reservoir states and distance sensors. The output of the SFA layer models the grid cells found in the entorhinal cortex of primates [6], similarly to the simulated rat's grid cells formed from visual input in [3]. The last layer is composed of 70 ICA units, which model place cells usually found in the CA areas of the hippocampus [6]. Grid cells are non-localized in the sense that they fire for more than a single location while place cells encode a specific position of the animal's environment.

As the robot has a very low speed, the input signal (17 distance sensors) is downsampled by a factor of $d_{t}=50$ (using the matlab function resample). Additionally, the leak rate in the reservoir is set to $\alpha=0.6$. These settings, optimized for the supervised scheme in [16], worked well for the current work (optimization and performance analysis of these parameters is left as future work). The matrix connecting the input to the reservoir $\left(\mathbf{W}_{\text {in }}\right)$ is initialized to $-0.2,0.2$ and 0 with probabilities $0.15,0.15$ and 0.7 , respectively.

The experiments are conducted using environment E1 (Fig. 1). It is a big maze with 64 predefined locations spread evenly around the environment (represented by small labeled triangles). First, for generating the input signal, the 
simulated robot navigates in the environment for 350.000 timesteps while its distance sensor measurements are recorded (the robot takes approximately 13.000 timesteps to visit most of the locations). The controller basically makes the robot explore the whole environment. After dowsampling the recorded input signal $\mathbf{u}(t)$, the number of samples becomes $n_{s}=7.000$. Next, the downsampled input signal is used to generate the reservoir states $\mathbf{x}(t), t=1,2, \ldots, n_{s}$ using (1).

The learning of the RC-SFA architecture takes place in 2 steps and uses $5 / 6$ of the input signal as the training dataset (1/6 for testing). First, the SFA layer learns by solving (6) where the inputs are the reservoir states and distance sensors (like in (7)). After $\mathbf{W}_{\mathrm{sfa}}$ is found, the output of SFA units $\mathbf{y}_{\mathrm{sfa}}(t), t=$ $1,2, \ldots, n_{s}$ is generated using (7). The second step corresponds to the learning of the upper ICA layer by applying the FastICA algorithm from Section 2.3 where the inputs for this layer are the output of the SFA units. The output signals $\mathbf{y}_{\text {sfa }}(t)$ and $\mathbf{y}_{\text {ica }}(t)$ are upsampled to the original sampling rate of $\mathbf{u}(t)$.

\section{$3.2 \quad$ Results}

The RC-SFA architecture is trained sequentially from the middle SFA layer to the top ICA layer. This section shows the results after training the layers with the input signal $\mathbf{u}(t)$ and reservoir states $\mathbf{x}(t)$ generated with the previously presented setup. Fig. 2(a) shows the output of 3 SFA units for a test input signal. The left plots show the outputs over time whereas the right plots show the response of the neurons as a function of the robot position in the environment. In the left plot, the horizontal axis represents the time, the left vertical axis denotes the real robot location (as given by the labeled triangles in Fig. 1), and the right vertical axis denotes the SFA output of the neuron. The colored dots represent the output of the SFA unit (where red denotes a peak response, green an intermediate response, and blue a low response). The SFA output is also shown as a black line in the same plot and as a colored trajectory in the right plot. As SFA units are ordered by slowness, the first SFA unit has the slowest response. It is high only for two areas of the environment: locations 10 to 17 , and locations 27 to 35 . Units 12 and 24 vary much faster, encoding several locations of the environment. In the same figure, it is possible to observe that SFA units learn a representation which is dependent on the robot heading. For instance, unit 12 responds differently for trajectories which go towards location 64 and trajectories that start from this position. As slow feature analysis is a method which is highly dependent on the input statistics, the movement patterns generated by the robot controller decisively influence the learning of the SFA units. In this context, most SFA units learn to be robot direction dependent in the current experiment. However, if the robot would change its direction more often (or faster compared to its speed), the SFA unit could learn to be direction invariant (by learning the slow features, that is, the robot position).

The upper ICA layer builds on the SFA layer. During learning, ICA units seek to maximize nongaussianity so that their responses become sparse and clustered and also as independent as possible. This form of sparse coding lead to the unsupervised formation of place cells. Fig. 2(b) shows a number of ICA units 
which code for specific locations in the environment. These units were chosen such that they code for adjacent locations as if the robot was navigating in the environment. The peak response is represented by white dots while lower responses are given gradually in darker colors. In order to view the localized aspect of place cells more clearly, the output of ICA units are ordered such that they have a spatial relationship. The reference locations (from 1 to 64), shown in environment E1 (Fig. 1), are used to automatically order the ICA layer. ICA units which do not respond strongly enough (that is, less than 4.5) in any situation are set to the end of the vector. Fig. 3(a) shows the real occupancy grid for the robot while it drives in environment E1 and the respective ICA activation map showing the spatially-ordered ICA responses (where $\mathbf{u}(t)$ is a test signal not used during learning). The peak responses are shown in black while white dots represent lower responses. Eleven ICA units (from 59 to 70) did not fire strongly enough and, so, did not code for any location. This activation map is very similar to the real robot occupancy grid showing that the place cells efficiently mapped most of the environment. Fig. 3(b) shows a magnification of the ICA activation map for locations under 20. It is possible to note that for almost the whole time period there is only a single ICA unit active (i.e., the ICA layer is detecting locations most of the time). This figure also clearly shows that most ICA units are dependent on the robot direction (as SFA units are). We have repeated the experiments shown here with the same datasets more than 15 times (where for each time a different random reservoir is created) with no visible changes in the learned place cells. Furthermore, preliminary results show that the RCSFA architecture also works for other kinds of environment configurations and environments with dynamic objects.

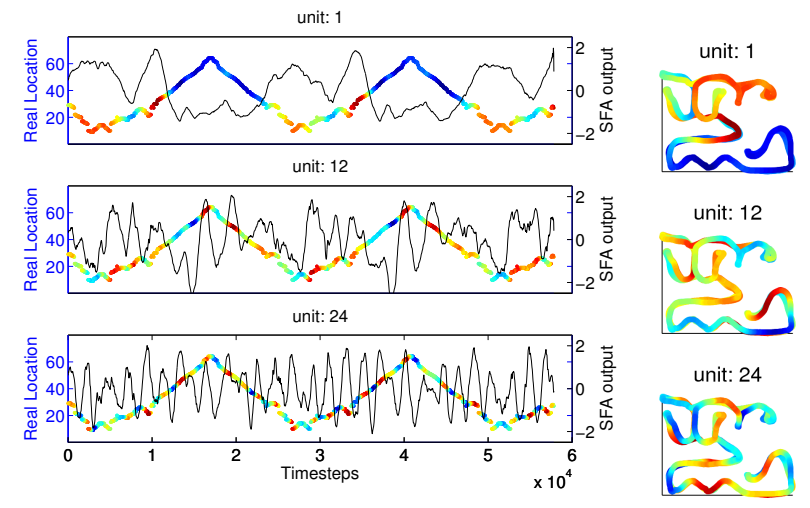

(a) SFA output

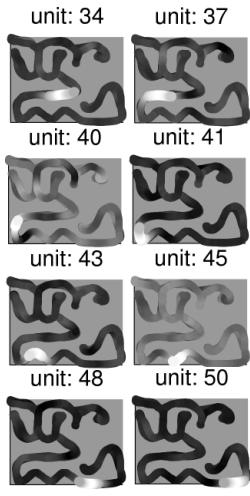

(b) ICA output

Fig. 2. Results for simulations in Environment E1. (a) Responses of SFA units 1, 12, and 24. Left: the SFA output over time. For each location (in time) given by the labeled triangles in Fig. 1, there is a colored dot where red denotes a peak response, green an intermediate response, and blue a low response. The output is also plot as a black line. Right: the same SFA output as a function of the robot position. (b) Response of ICA units as a function of the robot position. White dots denote high activity while darker dots represent lower responses. The results show the localized aspect of place cells or ICA units (the peak response is characteristic of one specific location). 


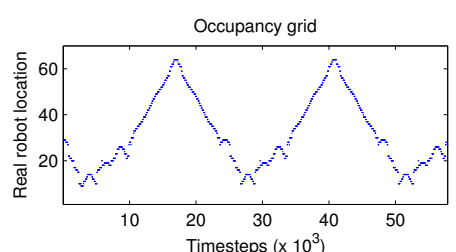

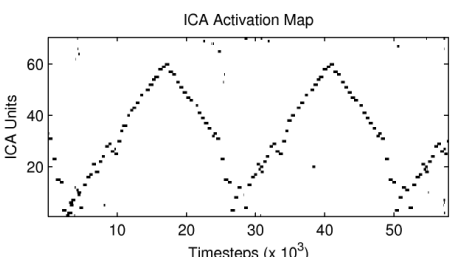

(a)

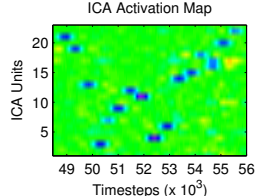

(b)

Fig. 3. Emergence of place cells in environment E1. (a) The real robot occupancy grid (left) and the respective spatially-ordered ICA activation map (black dots denote peak responses and white represent lower responses). (b) Close view of the ICA activation map where green denotes low response and blue high response.

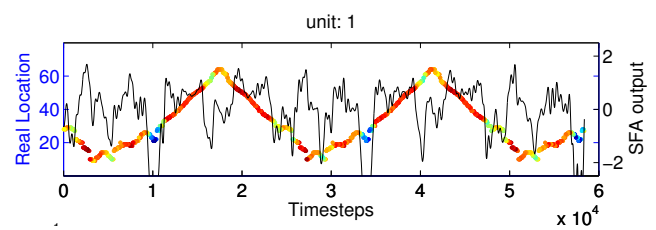

Fig. 4. Results for modified architecture without the reservoir layer in environment E1: the slowest SFA unit output over time, as in Fig. 2.

The importance of the reservoir can become more evident as it provides a short-term memory of previous inputs. In order to compare results, the proposed architecture in Fig. 1 is modified so that the inputs connect directly to the SFA layer and no reservoir is used at all. The following changes are also accomplished: the downsampling rate is increased to $d_{t}=200$ for slowing down the input signal; $n_{\text {sfa }}=100$ and $n_{\text {ica }}=100$. The SFA algorithm for this experiment includes a quadratic expansion process on the inputs [9], making the SFA effectively a non-linear process. The slowest SFA unit is shown in Fig. 4. The response pattern from this unit seems more noisy than the case when using the RC-SFA architecture which shows a smooth signal (Fig. 2(a)). The ICA activation map for this architecture (not shown) was very fuzzy and far from the one obtained in Fig. 3 (also did not solve the perceptual aliasing problem once one ICA unit coded for multiple similar but distinct locations). In this way, this modified setup without the reservoir was not able to model place cells in the current experiment.

\section{Conclusion and Future Work}

This work proposes a new biologically inspired architecture (RC-SFA) based on a mixture of recently developed techniques (Reservoir computing, Slow Feature Analysis and Independent Component Analysis). The RC-SFA is a general architecture which can be easily applied to wide range of applications (e.g., extract slowly-varying components of an input signal such as: behaviors or movements of a humanoid robot or phonemes and words from speech data). In this work, a simulated mobile robot (with few proximity sensors) autonomously learns, using our proposed RC-SFA architecture, a rather accurate spatial representation of its environment and, in this way, to self-localize in it. We do not use proprioceptive information as a form of spatial memory, but rather the short-term memory 
of the reservoir has shown to eliminate the perceptual aliasing from sensors, producing a system which autonomously learns the relevant information from the input stream. Further interesting directions for research include the validation of the proposed architecture with a real robot (such as the e-puck robot) and accomplish further experiments such as kidnapping the robot and navigation in a dynamic environment. Other important questions include: how distinct settings of reservoir parameters influence the learning of the spatial representation; what kind of environments can be used; and what range of noise and sensor failures the model can cope with.

\section{References}

1. Thrun, S., Burgard, W., Fox, D.: Probabilistic Robotics. The MIT Press (2005)

2. Arleo, A., Smeraldi, F., Gerstner, W.: Cognitive navigation based on nonuniform gabor space sampling, unsupervised growing networks, and reinforcement learning. IEEE Transactions on Neural Networks 15(3) (May 2004) 639-652

3. Franzius, M., Sprekeler, H., Wiskott, L.: Slowness and sparseness lead to place, head-direction, and spatial-view cells. PLoS Comput. Biol. 3(8) (2007) 1605-1622

4. Stroesslin, T., Sheynikhovich, D., Chavarriaga, R., Gerstner, W.: Robust selflocalisation and navigation based on hippocampal place cells. Neural Networks 18(9) (2005) 1125-1140

5. Chavarriaga, R., Strsslin, T., Sheynikhovich, D., Gerstner, W.: A computational model of parallel navigation systems in rodents. Neuroinformatics 3 (2005) 223-241

6. Moser, E.I., Kropff, E., Moser, M.B.: Place cells, grid cells and the brains spatial representation system. Annual Reviews of Neuroscience 31 (2008) 69-89

7. Schrauwen, B., Verstraeten, D., Van Campenhout, J.: An overview of reservoir computing: theory, applications and implementations. In: Proc. of ESANN. (2007)

8. Jaeger, H.: The "echo state" approach to analysing and training recurrent neural networks. Technical Report GMD Report 148, German National Research Center for Information Technology (2001)

9. Wiskott, L., Sejnowski, T.J.: Slow feature analysis: Unsupervised learning of invariances. Neural Computation 14(4) (2002) 715-770

10. Hyvärinen, A., Oja, E.: Independent component analysis: algorithms and applications. Neural Networks 13 (2000) 411-430

11. Maass, W., Natschläger, T., Markram, H.: Real-time computing without stable states: A new framework for neural computation based on perturbations. Neural Computation 14(11) (2002) 2531-2560

12. Yamazaki, T., Tanaka, S.: The cerebellum as a liquid state machine. Neural Networks 20 (2007) 290-297

13. Jaeger, H., Lukosevicius, M., Popovici, D.: Optimization and applications of echo state networks with leaky integrator neurons. Neural Networks 20 (2007) 335-352

14. Berkes, P., Wiskott, L.: Slow feature analysis yields a rich repertoire of complex cell properties. Journal of Vision 5 (2005) 579-602

15. Antonelo, E.A., Baerlvedt, A.J., Rognvaldsson, T., Figueiredo, M.: Modular neural network and classical reinforcement learning for autonomous robot navigation: Inhibiting undesirable behaviors. In: Proceedings of IJCNN, Vancouver, Canada (2006) 498- 505

16. Antonelo, E.A., Schrauwen, B., Stroobandt, D.: Event detection and localization for small mobile robots using reservoir computing. Neural Networks 21 (2008) $862-871$ 